\title{
Relationship of the balloon analog risk task to neurocognitive impairment differs by HIV serostatus and history of major depressive disorder
}

\author{
Rowan Saloner ${ }^{1,2,3}$ (1) Erin E. Morgan ${ }^{2} \cdot$ Mariam A. Hussain ${ }^{1,2} \cdot$ David J. Moore $^{2} \cdot$ Robert K. Heaton $^{2} \cdot$ \\ Mariana Cherner ${ }^{2} \cdot$ Igor Grant $^{2} \cdot$ Jennifer E. ludicello ${ }^{2} \cdot$ the TMARC Group $^{2}$
}

Received: 29 June 2021 / Revised: 11 November 2021 / Accepted: 16 December 2021 / Published online: 3 January 2022

(c) The Author(s) 2021

\begin{abstract}
HIV and major depressive disorder (MDD) commonly co-occur and are both linked to greater risk-taking behavior, possibly due to neurocognitive impairment (NCI). The present study examined the concordance of the Balloon Analog Risk Task (BART), a gold standard measure of risk-taking propensity, with NCI and real-world sexual risk behaviors in PWH with comorbid MDD. Participants included 259 adults, stratified by HIV serostatus (HIV +/HIV -) and lifetime MDD (MDD +/MDD -), who completed neuropsychological testing, the BART, and sexual risk behavior questionnaires. Logistic regression, stratified by HIV serostatus, examined joint effects of MDD and BART (linear and quadratic) on NCI. Followup linear regressions examined sexual risk behavior and neurocognitive domain T-scores as correlates of the BART. NCI prevalence was lowest in HIV - /MDD - , but BART scores did not differ by HIV/MDD status. In the HIV + group, BART performance predicted NCI such that high and low BART scores related to greater odds of NCI, but only in dual-risk HIV + $/$ MDD + individuals. HIV +/MDD + individuals with both low and high BART scores exhibited poorer learning and recall, whereas processing speed and executive function were only poor in low BART risk-taking HIV +/MDD + . Higher BART scores linearly related to higher sexual risk behaviors only in MDD + individuals, independent of HIV serostatus. Low and high risk-taking on the BART may reflect discrete neurocognitive profiles in HIV +/MDD + individuals, with differential implications for real-world sexual risk behavior. HIV and comorbid MDD may disturb corticostriatal circuits responsible for integrating affective and neurocognitive components of decision-making, thereby contributing to risk-averse and risktaking phenotypes.
\end{abstract}

Keywords HIV-associated neurocognitive disorders $\cdot$ Depression $\cdot$ Risk-taking $\cdot$ Cognition $\cdot$ HIV risk $\cdot$ Decision making

Rowan Saloner

rsaloner@ucsd.edu

1 Joint Doctoral Program in Clinical Psychology, San Diego State University/University of California, San Diego, San Diego, CA, USA

2 Department of Psychiatry, HIV Neurobehavioral Research Program, University of California, San Diego, San Diego, CA, USA

3 Department of Neurology, Memory and Aging Center, University of California, San Francisco, San Francisco, CA, USA

\section{Introduction}

HIV disease is no longer considered a terminal illness due to effective antiretroviral therapy (ART). Accordingly, the clinical care for persons with HIV (PWH) has now shifted toward adherence to ART, treatment of HIV-related comorbidities, and monitoring of behaviors that increase risk for HIV transmission. Early in the course of the disease, HIV is capable of infiltrating the central nervous system (CNS) and producing neuroimmunological insults, particularly in the presence of ART regimens that have poor CNS penetration (Ellis et al. 2007; Hult et al. 2008). Although the neuroinflammatory cascades that drive HIV neuropathologenesis can diffusely impact the CNS, frontostriatal circuits that support higherorder neurocognitive functions, emotional regulation, and reward processing are particularly vulnerable to HIV-related 
neural injury (Soontornniyomkij et al. 2016; Woods et al. 2009). Roughly $30-50 \%$ of PWH present with neurocognitive impairment (NCI; Heaton et al. 2010; Saloner and Cysique 2017). In addition to premorbid psychosocial risk factors that precede the acquisition of HIV, acquired frontostriatal injury due to HIV is thought to contribute to the high prevalence of NCI and neuropsychiatric disturbances, including major depressive disorder (MDD) and substance use disorders (Anand et al. 2010; Arseniou et al. 2014; Ipser et al. 2015). An estimated $22-54 \%$ of PWH meet clinical criteria for a lifetime diagnosis of MDD (Rabkin 2008; Rooney et al. 2019; Rubin and Maki 2019), which can impact real-world outcomes such as ART adherence and employment (Heaton et al. 2004a; Rabkin 2008).

Group level comparisons on standard neuropsychological tests between PWH and HIV-seronegative (HIV -) counterparts often demonstrate HIV-associated deficits in episodic memory (typically mixed encoding and retrieval profile), cognitive and psychomotor slowing, poor complex attention/working memory, and executive dysfunction (including novel problem solving and set-shifting; Woods et al. 2009). Recent studies have also begun to incorporate experimental cognitive neuropsychology paradigms into neurobehavioral assessment protocols to tap into multifaceted constructs that more closely resemble real-world situations than traditional clinical neuropsychological measures (Llewellyn 2008; Woods et al. 2009). Risk-taking is one such construct that has received considerable attention, given that the likelihood of acquiring or transmitting HIV is heightened by the decision to engage in risky behaviors such as unprotected sex and injection drug use (Crooks et al. 2015; Iudicello et al. 2013; Montoya et al. 2016).

The Iowa Gambling Task (IGT; Bechara 2007; Bechara et al. 1994) is the most commonly studied experimental measure of risky decision-making in PWH. The IGT requires participants to choose from one of four card decks, with two disadvantageous "risky" decks reflecting larger, immediate rewards but higher long-term penalties and two advantageous "safe" decks reflecting smaller, immediate rewards but higher long-term earnings. Studies examining the neuropsychological correlates of IGT performance in PWH with and without comorbid substance use diagnoses demonstrate "riskier" decision-making in PWH, particularly among individuals with deficits in inhibitory control, learning, and memory (Hardy et al. 2006; Iudicello et al. 2013; Martin et al. 2013; Martin et al. 2004). Notably, several studies have demonstrated that the concordance of the IGT with standard neuropsychological measures and real-world risk behaviors is influenced by affective factors. Thames and colleagues demonstrated a mediating effect of depression on the relationship between executive function and IGT performance in PWH (Thames et al. 2012), while several studies have identified moderating effects of emotional distress and sensation seeking personality traits on the relationship between IGT performance and sexual risk behaviors and substance use in PWH (Golub et al. 2016; Gonzalez et al. 2005; Wardle et al. 2010).

The Balloon Analog Risk Task (BART) is another common laboratory-based assessment of risk-taking that requires participants to make sequential decisions about whether to progressively pump up a balloon to earn money or stop pumping and collect their earnings (Lejuez et al. 2002). Each sequential pump results in a fixed and known monetary gain but also an increase in the unknown probability that the balloon explodes, which would result in the loss of previously accrued monetary gains. The BART is less dependent upon an individual's ability to learn reward and punishment contingencies than the IGT and is therefore considered a more direct measure of risk-taking propensity (Hevey et al. 2017; Llewellyn 2008), which is defined as the tendency to engage in behaviors that may yield positive outcomes but also carry an uncertain likelihood of negative outcomes (Balogh et al. 2013; Kreek et al. 2005). Participants do not have to learn which behaviors are risky - they are explicitly told each pump will result in a fixed gain or balloon pop and therefore the BART is more reflective of real world risktaking in which individuals willingly engage in behaviors that expose themselves to potential punishment in the pursuit of rewards (Bishara et al. 2009). The average number of pumps on unexploded balloons is the primary score used to measure BART performance (i.e., risk-taking propensity), and this metric has been found to converge with real-world risk behaviors (e.g., substance use, unprotected sex; Hunt et al. 2005; Lejuez et al. 2002, 2004).

Both low and high pumps may indicate suboptimal BART performance, but due to different mechanisms. Several studies have reported significantly higher BART pumping tendencies in PWH compared to HIV- controls, which has been interpreted as a greater propensity toward risk-taking (Meade et al. 2016; Paydary et al. 2016). In these studies, poorer performance (i.e., higher average number of pumps) was linked to higher levels of impulsivity (Paydary et al. 2016) and altered functioning in brain regions affected by HIV and linked to decision-making (e.g., prefrontal cortex and anterior cingulate; Meade et al. 2016). However, studies in populations with acquired brain injuries, including those in which risk-taking and poor decision-making are clinical hallmarks (e.g., frontotemporal dementia), frequently observe fewer pumps in patients compared to healthy controls (Balagueró et al. 2016; Fecteau et al. 2013; Strenziok et al. 2011). Although lower BART pumps reduces the likelihood of a balloon explosion, these observations in patients with NCI are interpreted as impaired stimulus-reinforcement learning, given that lower risk-taking will also reduce potential earnings. Research from computational cognitive models also suggests that lower BART pumps may manifest from greater perceived probability 
of losing (loss sensitivity) and less consistent decision-making (Bishara et al. 2009; Kahneman and Tversky 1979).

Despite being considered a gold-standard measure of risk-taking propensity, little is known about the concordance of the BART with standard neuropsychological performance and HIV transmission risk behaviors in PWH, as well as the moderating role of depression on these relationships. Moreover, the majority of studies have assumed linear associations between the BART and other aspects of neurobehavior, despite evidence that risk aversion and risk-taking can both be indicative of neurocognitive and affective dysfunction (James et al. 2015; Smoski et al. 2008; Whittle et al. 2015). The present study leveraged comprehensive neurobehavioral data from a cohort study of PWH and HIV-individuals to evaluate linear and non-linear relationships of the BART with NCI and HIV transmission risk behaviors. Moreover, we examined whether these relationships were moderated by depression, defined by clinical diagnoses of lifetime MDD. Consistent with the existing literature, we hypothesized that both low and high levels of risk-taking propensity (i.e., BART performance) would relate to higher odds of NCI compared to intermediate levels of risk-taking propensity, and that higher levels of risk-taking propensity would be associated with increased HIV transmission risk behaviors. In addition, we hypothesized that the relationships between risk-taking propensity, NCI, and HIV transmission risk behaviors would be strongest in PWH with MDD reflecting greater disturbance in the cognitive and affective components of risk taking and decision-making.

\section{Materials and methods}

\section{Participants}

Participants were 131 HIV-seropositive (HIV +) and 128 HIV - adults enrolled in the University of California San Diego's (UCSD) Translational Methamphetamine AIDS Research Center (TMARC), a NIDA-funded cohort study focusing on the effects of HIV and methamphetamine (METH) on neurobehavioral functioning. Given the present study's focus on the relationship between risk-taking and neurocognition in the context of HIV and depression, METH use disorder (defined by TMARC as a history of METH dependence with abuse or dependence within the past 18 months, as diagnosed by the Composite International Diagnostic Interview [CIDI] (World Health Organization 1998)) was considered as a covariate while participants were stratified by HIV and lifetime MDD diagnoses into four groups: HIV - /MDD - $(n=92), \mathrm{HIV}-/ \mathrm{MDD}+(n=36)$, $\mathrm{HIV}+/ \mathrm{MDD}-(n=75)$, and HIV +/MDD + $(n=56)$. Participants provided written informed consent to study procedures, which were approved by the UCSD Institutional
Review Board. Potential participants were excluded if they reported a history of a psychotic or mood disorder with psychotic features, or had a neurological (e.g., stroke, seizure disorder) or non-HIV medical condition (e.g., hepatitis C) that may confound neurobehavioral test results. Given the overarching cohort study aims, TMARC criteria excluded participants with recent alcohol dependence (within the last 12 months) or recent diagnoses of abuse (within the last 12 months) or dependence (within the last 5 years) for all other substances except METH and cannabis.

\section{Neuromedical assessment}

All participants underwent a comprehensive neuromedical assessment, blood draw, and lumbar puncture. HIV disease was diagnosed by enzyme-linked immunosorbent assay (ELISA) with Western blot confirmation. Among PWH, plasma HIV RNA was measured using reverse transcriptasepolymerase chain reaction (Amplicor, Roche Diagnostics, Indianapolis, IN) and deemed undetectable at a lower limit of quantitation (LLQ) of 50 copies $/ \mathrm{ml}$.

\section{Neuropsychiatric assessment}

To determine if participants had experienced a clinically significant history of depressed mood, the CIDI (World Health Organization 1998) was administered by trained psychometrists to establish DSM-IV lifetime and current (within the last 30 days) diagnoses of MDD. For the present analysis, participants who met criteria for a lifetime MDD diagnosis were grouped as MDD+, given that few participants met criteria for current MDD $(n=15)$. The CIDI was also used to diagnose lifetime and current substance use disorders and Antisocial Personality Disorder.

Participants also completed several self-report measures to characterize recent levels of depressive symptomatology in the MDD + and MDD - groups. Specifically, the total score from the second edition of the Beck Depression Inventory-II (BDI-II; Beck et al. 1996) was used to measure the severity of overall symptoms of depression over the 2 weeks prior to the study visit. Consistent with the methodology reported by Marquine et al. (2014), a composite apathy score was also generated by combining apathy-related items from the BDIII (i.e., loss of pleasure, loss of interest, difficulty making decisions, and feelings of tiredness and fatigue), the "after illness" apathy subscale from the Frontal Systems Behavior Scale (FrSBe; Grace, 2001), and the vigor-activity subscale from the Profile of Mood States (McNair 1992). Raw scores on the individual subscales were converted to z-scores based on the mean and standard deviation of the entire TMARC study healthy control group (i.e., HIV-/METH-), and z-scores were then averaged and converted to T-scores. Higher apathy $\mathrm{T}$-scores represent higher levels of apathy. 
Using the same composite approach, T-scores were also derived for non-depressive "frontal systems" traits of impulsivity/disinhibition and sensation-seeking (Marquine et al. 2014), which have been shown to influence risky decision-making in PWH (Gonzalez et al. 2005; Paydary et al. 2016; Wardle et al. 2010). Impulsivity/disinhibition T-scores were derived from the 'after illness' disinhibition subscale from the FrSBe, the urgency and lack of premeditation subscales from the UPPS Impulsive Behavior Scale (Whiteside and Lynam 2001), and the Barratt Impulsiveness Scale total score (Patton et al. 1995). Sensationseeking T-scores were derived from the Kalichman sexual and non-sexual sensation-seeking scales (Kalichman et al. 1994; Kalichman and Rompa 1995). Higher T-scores represent greater impulsivity/disinhibition or sensation-seeking traits.

\section{Neurobehavioral assessment}

\section{Neuropsychological testing}

All participants completed a comprehensive and standardized neuropsychological assessment including an estimate of premorbid verbal IQ (i.e., Wide Range Achievement Test Reading subtest, Version 4 (Wilkinson and Robertson 2006) and seven neurocognitive domains commonly impacted by HIV (Heaton et al. 2010; Morgan et al. 2012; Rippeth et al. 2004). The domains and individual tests were: verbal fluency (Controlled Oral Word Association Test, animal fluency, action fluency), processing speed (Trail Making Test A, WAIS-III Digit Symbol, WAIS-III Symbol Search, Stroop Color and Word Test Color Score), executive function (Wisconsin Card Sorting Test-64 Card Version, Trail Making Test B, Stroop Color and Word Test Interference Score), learning and delayed recall (Hopkins Verbal Learning Test-Revised, Brief Visuospatial Memory Test-Revised), working memory (WMS-III Spatial Span, Paced Auditory Serial Addition Test), and complex motor skills (Grooved Pegboard Test). For participants who had been exposed to the test battery at prior research visits, raw scores for each test were converted to practice effect-adjusted scaled scores (Cysique et al. 2011). Using the most comprehensive normative standards available, scaled scores were converted to demographically-corrected T-scores that adjusted for the effects of age, education, sex, and race/ethnicity, as appropriate (Heaton et al. 2004b, 2003; Norman et al. 2011). Individual test $\mathrm{T}$-scores were averaged within each domain to derive domain-specific T-scores, which were examined as secondary outcomes in the present study. In order to classify neurocognitive impairment, the primary outcome of interest, T-scores were converted to deficit scores that give differential weight to impaired, as opposed to normal scores, on a scale ranging from $0(T \geq 40$; normal) to $5(T<20$; severe impairment). Deficit scores were averaged across the entire test battery to derive a global deficit score (GDS). Consistent with prior studies, the presence of global neurocognitive impairment was classified as GDS $\geq 0.5$. (Blackstone et al. 2012; Carey et al. 2004).

\section{Balloon analog risk task}

The BART is a computer-simulated measure of risky decision making that has strong convergent validity with realworld risk behaviors and self-report trait-measures of risktaking tendencies (Hunt et al. 2005; Lejuez et al. 2002). The present study employed the same BART paradigm as described in detail by Hunt et al. (2005). Briefly, participants have the opportunity to earn simulated money by clicking a balloon pump button that inflates a simulated balloon presented on the computer screen. Participants earn 1 cent of simulated money per balloon pump. However, they are informed that the balloon will explode at some point, that the exact explosion point will vary across trials, and that they will lose money accrued on a trial if the balloon explodes. Thus, each trial ends when 1) the participant decides to stop pumping and collect money or 2) the balloon explodes. The maximum number of possible pumps for any given trial is 128 and the probability of explosion, $x$, on a given pump, $i$, is $x=1 /[(128-i)+1)]$. Thus, each additional pump is associated with a higher likelihood of losing financial gains as well as a decrease in the relative gain to be earned (i.e., diminished rewards). Participants completed 30 trials and the explosion point on each specific trial was the same across all participants. At the end of the study visit, participants were awarded actual money (up to \$15) that was proportional to the simulated money they earned on the BART. The average number of balloon pumps on trials in which the balloon did not explode was used as the primary predictor for analyses (i.e., "BART adjusted pumps"). The adjusted pumps value is the most commonly used index of risk-taking propensity on the BART because it is not confounded by the fact that explosion points vary across balloon trials (Lauriola et al. 2014). The total number of trials with explosions is also provided as an additional metric for descriptive purposes.

\section{HIV transmission risk behavior T-scores}

In order to capture real-world risky decision-making that holds clinical relevance, participants completed questionnaires assessing for risky sexual behaviors: (1) Sexual Risk Scale subscore from the Modified Risk Assessment Battery (RAB; Navaline et al. 1994), and (2) Sexual Risk Scale total score (SRS; DeHart and Birkimer 1997). On the Modified RAB Sexual Risk Scale, participants rate engagement in sexual risk behaviors (e.g., frequency of unprotected sex) over 
the past 6 months, with higher scores representing greater sexual risk-taking. The SRS measures attitudes, norms, intention, and expectations related to practicing safer sex, with higher total scores suggesting a stronger intention to practice safe sex. For ease of interpretation, the total SRS score was reverse-coded to align with the RAB such that higher scores represented riskier intentions. Employing the same methodology as the "frontal systems" behaviors T-scores approach described above (Marquine et al. 2014), RAB and SRS scores were converted to z-scores based on the entire TMARC study control group and then averaged to form a composite HIV transmission risk behaviors T-score. Higher T-scores represent a greater propensity to engage in risky sexual behaviors that enhance risk for HIV acquisition and transmission. HIV transmission risk behaviors T-scores were used as a secondary outcome in analyses examining the ecological validity of the BART risk-taking index.

\section{Statistical analysis}

HIV/MDD group comparisons on demographic, psychiatric, substance use, HIV disease characteristics (HIV + stratum only), and neurobehavioral variables were performed with ANOVA, Wilcoxon rank-sum tests, or likelihood ratio $\chi^{2}$ tests, as appropriate. Stepwise multivariable logistic regression models that examined the interactive effects of MDD and risk-taking (i.e., BART adjusted pumps) on odds of NCI were conducted separately for each HIV stratum. Variables that demonstrated univariable trend-level associations $(p<0.10)$ with the independent variables (MDD group or BART adjusted pumps) or the primary dependent variable (NCI) were entered as covariates in step 1. Depression and apathy scales (i.e., BDI-II score and apathy T-scores) were reported to further characterize the clinical MDD groups, but were not considered as candidate covariates given the high degree of overlap with the construct of depression captured by the clinical MDD groups. The optimal combination of covariate predictors in step 1 were determined based on Akaike information criteria (AIC) with backwards selection (Akaike, 1974; Burnham and Anderson 2004). The main effect of MDD group and the linear and quadratic effects of BART adjusted pumps were entered in step 2. If nonsignificant $(p<0.05)$, the quadratic adjusted pumps term was removed in step 2 and the model was re-run to obtain an appropriate estimate of the linear adjusted pumps term. Last, the interaction(s) between MDD group and the BART adjusted pumps term(s) were entered in step 3. This stepwise approach allowed us to determine the incremental contributions of the independent main effects and interactions of interest above and beyond the contributions of relevant covariates. These stepwise regression analyses were stratified by HIV serostatus given the limited power to detect a 4-way interaction effect (i.e., HIV $\times$ MDD $\times$ [adjusted pumps $\times$ adjusted pumps]). Nagelkerke pseudo- $R^{2}$ goodness-of-fit statistics for a categorical dependent variable (i.e., NCI) are reported to facilitate comparisons between the nested stepwise logistic regression models (Nagelkerke 1991). Odds ratios (OR) are reported as effect size estimates for individual parameters in logistic regression analyses.

To determine which neurocognitive domains were driving any significant interactions of MDD and BART adjusted pumps on the global NCI classification, follow-up linear regression analyses examined the seven neurocognitive domain-specific T-scores as separate outcomes. In order to limit the Type I error rate for these seven additional analyses, we used the false discovery rate (FDR) method and set the FDR at 5\% (Benjamini and Hochberg 1995). Last, to determine the ecological validity of the laboratory-based BART index of risk-taking propensity for clinically-relevant realworld risk behaviors, we examined relationships between BART adjusted pumps and HIV transmission risk behavior T-scores across the entire study sample and within the four study groups. All analyses were conducted using JMP Pro version 14.0.0 (JMP ${ }^{\circledR}$, Version $<12.0 .1>$. SAS Institute Inc., Cary, NC, 2018).

\section{Results}

\section{Study group characteristics}

The full study sample was $56 \%$ non-Hispanic White and $81 \%$ male with a mean age of 41.8 years and mean education of 13.8 years. Participant characteristics by MDD group, stratified by HIV serostatus, are presented in Table 1 . Age, sex, years of education, estimated premorbid verbal IQ, and race/ethnicity were comparable between MDDand MDD + groups in both HIV strata $(p s>0.069)$. In the HIV - stratum, MDD + participants exhibited higher rates of METH use disorders $(\mathrm{OR}=5.12, p<0.001)$ and lifetime non-METH use disorders $(\mathrm{OR}=3.33, p=0.011)$ than MDD- participants; substance use characteristics did not significantly differ by MDD group in the HIV + stratum ( $p$ s $>0.140$ ). Similarly, HIV disease characteristics did not differ by MDD group in the HIV + stratum ( $p$ s $>0.240)$, with the majority of participants on ART medication (81\%) and virally suppressed $(70 \%)$.

MDD + participants expectedly had higher (but still overall low) rates of current MDD (HIV - : $11 \%, \mathrm{HIV}+: 20 \%$ ) and higher BDI-II (Cohen's $d=0.55, p<0.001)$ and apathy scores $(d=0.48, p<0.001)$ than MDD - participants, regardless of HIV serostatus. Overall scores on the scales measuring recent depressive symptoms were significantly higher in HIV + participants compared to HIV - participants (BDI-II: $d=0.39, p=0.002$; apathy: $d=0.53, p<0.001)$. Although the HIV +/MDD - group by definition had never met criteria 
Table 1 Study group characteristics

\begin{tabular}{|c|c|c|c|c|c|c|}
\hline \multirow[t]{2}{*}{ Variable } & \multicolumn{3}{|l|}{ HIV - } & \multicolumn{3}{|l|}{ HIV + } \\
\hline & $\operatorname{MDD}-(n=92)$ & $\mathrm{MDD}+(n=36)$ & $p$ & $\operatorname{MDD}-(n=75)$ & $\mathrm{MDD}+(n=56)$ & $p$ \\
\hline \multicolumn{7}{|l|}{ Demographics } \\
\hline Age (years) & $41.1(14.5)$ & $39.6(13.6)$ & .573 & $42.3(13.4)$ & $43.9(11.1)$ & .467 \\
\hline Sex (male) & $63(68.5 \%)$ & $23(63.9 \%)$ & .773 & $71(94.7 \%)$ & $52(92.9 \%)$ & .953 \\
\hline Education (years) & $13.7(2.5)$ & $12.8(2.5)$ & .070 & $14.2(2.5)$ & $14.1(2.3)$ & .844 \\
\hline Estimated premorbid verbal IQ & $102.2(13.0)$ & $100.2(14.5)$ & .451 & $102.0(13.2)$ & $104.3(10.3)$ & .284 \\
\hline Race/ethnicity & & & .752 & & & .142 \\
\hline Non-Hispanic White & $50(54.3 \%)$ & $21(58.3 \%)$ & & $38(50.7 \%)$ & $36(64.3 \%)$ & \\
\hline Black & $14(15.2 \%)$ & $6(16.7 \%)$ & & $12(16.0 \%)$ & $7(12.5 \%)$ & \\
\hline Hispanic & $21(22.8 \%)$ & $6(16.7 \%)$ & & $23(30.7 \%)$ & $9(16.1 \%)$ & \\
\hline Asian & $2(2.2 \%)$ & $2(5.6 \%)$ & & $0(0.0 \%)$ & $2(3.6 \%)$ & \\
\hline Other & $5(5.4 \%)$ & $1(2.8 \%)$ & & $2(2.7 \%)$ & $2(3.6 \%)$ & \\
\hline \multicolumn{7}{|l|}{ Depression characteristics } \\
\hline Current major depressive disorder & $0(0.0 \%)$ & $4(11.1 \%)$ & .007 & $0(0.0 \%)$ & $11(19.6 \%)$ & $<.001$ \\
\hline BDI-II score & $3[0,13]$ & $6[3,20]$ & .021 & $7[2,15]$ & $15[5,26]$ & .001 \\
\hline Apathy T & $56.4(14.7)$ & $62.3(16.7)$ & .052 & $63.9(19.3)$ & $72.3(18.7)$ & .018 \\
\hline \multicolumn{7}{|l|}{ Frontal systems behaviors } \\
\hline Impulsivity/disinhibition $\mathrm{T}$ & $56.8(15.0)$ & $60.7(14.7)$ & .186 & $59.2(14.2)$ & $59.8(11.8)$ & .802 \\
\hline Sensation-seeking behaviors $\mathrm{T}$ & $51.9(11.0)$ & $49.2(8.0)$ & .189 & $53.8(9.8)$ & $52.7(9.6)$ & .518 \\
\hline ASPD & $16(17.6 \%)$ & $11(30.6 \%)$ & .116 & $9(12.0 \%)$ & $10(17.9 \%)$ & .349 \\
\hline \multicolumn{7}{|l|}{ Alcohol and substance use } \\
\hline METH use disorder & $31(33.7 \%)$ & $26(72.2 \%)$ & $<.001$ & $33(44.0 \%)$ & $26(46.4 \%)$ & .921 \\
\hline Lifetime non-METH SUD & $12(13.0 \%)$ & $12(33.3 \%)$ & .011 & $10(13.3 \%)$ & $5(8.9 \%)$ & .428 \\
\hline Lifetime alcohol use disorder & $39(42.4 \%)$ & $21(58.3 \%)$ & .104 & $35(46.7 \%)$ & $19(33.9 \%)$ & .141 \\
\hline Tobacco smoking history & & & .292 & & & 623 \\
\hline Current & $20(21.7 \%)$ & $7(19.4 \%)$ & & $16(21.3 \%)$ & $16(28.6 \%)$ & \\
\hline Past & $43(46.7 \%)$ & $22(61.1 \%)$ & & $38(50.7 \%)$ & $25(44.6 \%)$ & \\
\hline Never & $29(31.5 \%)$ & $7(19.4 \%)$ & & $21(28.0 \%)$ & $15(26.8 \%)$ & \\
\hline \multicolumn{7}{|l|}{ HIV disease characteristics } \\
\hline AIDS diagnosis & & & & $33(44.0 \%)$ & $25(44.6 \%)$ & .942 \\
\hline Duration of HIV infection (years) & & & & $6.7[1.6,15.2]$ & $7.5[2.4,17.4]$ & .550 \\
\hline Nadir CD4 count (cells/mm³) & & & & $250[107,373]$ & $300[150,443]$ & .241 \\
\hline Current CD4 count (cells/mm $\mathrm{mm}^{3}$ ) & & & & $591[344,783]$ & $565[467,793]$ & .289 \\
\hline Detectable plasma viral load & & & & $19(26.4 \%)$ & $20(35.7 \%)$ & .257 \\
\hline On ART & & & & $62(82.7 \%)$ & $44(78.6 \%)$ & .556 \\
\hline
\end{tabular}

Values are presented as mean (SD), median [IQR], or $n(\%)$

$A R T$ antiretroviral therapy, ASPD antisocial personality disorder, BDI-II Beck Depression Inventory version two, SUD substance use disorder

for a lifetime (or current) MDD diagnosis, this group had similar BDI-II and apathy scores compared to the HIV - / $\mathrm{MDD}+\operatorname{group}(p s>0.480)$.

\section{Neurobehavioral performance by HIV/MDD group}

Table 2 reports performance on neuropsychological testing, the BART, and HIV transmission risk behaviors across HIV/ MDD groups. The prevalence of global NCI was $31 \%$ in the full study sample. A one-way likelihood ratio $\chi^{2}$ test indicated a trend-level omnibus difference in the prevalence of global
$\mathrm{NCI}$ across the four groups $\left(\chi^{2}=6.90, p=0.075\right)$. Compared to HIV - /MDD - (21\%), the odds of NCI were roughly twice as high in the three risk groups: HIV - /MDD + $(36 \%$, $\mathrm{OR}=2.17, p=0.073)$, HIV $+/ \mathrm{MDD}-(35 \%, \mathrm{OR}=2.04$, $p=0.044), \mathrm{HIV}+/ \mathrm{MDD}+(38 \%, \mathrm{OR}=2.31, p=0.027)$. Rates of NCI did not differ between the three risk groups (OR range: 0.94 to 1.13 ). A similar pattern was generally observed for domain-specific performance (refer to Table 2). Significant omnibus HIV/MDD effects were detected for processing speed $\left(F=3.45, p=0.017, \eta^{2}=0.039\right)$ and executive functioning T-scores $\left(F=4.30, p=0.006, \eta^{2}=0.048\right)$, 
Table 2 Neurocognitive performance and risk-taking by HIV and MDD group

\begin{tabular}{|c|c|c|c|c|c|}
\hline Variable & $\begin{array}{l}\mathrm{HIV}-/ \\
\mathrm{MDD}-(n=92)\end{array}$ & $\begin{array}{l}\mathrm{HIV}-/ \\
\mathrm{MDD}+(n=36)\end{array}$ & $\begin{array}{l}\mathrm{HIV}+/ \\
\mathrm{MDD}-(n=75)\end{array}$ & $\begin{array}{l}\mathrm{HIV}+/ \\
\mathrm{MDD}+(n=56)\end{array}$ & $p$ \\
\hline \multicolumn{6}{|l|}{ Neuropsychological testing } \\
\hline Global neurocognitive impairment & $19(20.7 \%)$ & $13(36.1 \%)$ & $26(34.7 \%)$ & $21(37.5 \%)$ & .075 \\
\hline Verbal fluency $\mathrm{T}$ & $50.2(8.3)$ & $50.6(8.6)$ & $47.2(8.1)$ & $49.2(7.9)$ & .079 \\
\hline Processing speed $\mathrm{T}^{\mathrm{a}}$ & $51.6(8.5)$ & $49.3(8.4)$ & $48.0(7.0)$ & $48.2(8.0)$ & .017 \\
\hline Executive functioning $\mathrm{T}^{\mathrm{b}}$ & $51.2(8.9)$ & $47.3(9.2)$ & $47.5(8.1)$ & $46.8(8.7)$ & .006 \\
\hline Learning $\mathrm{T}$ & $44.5(8.0)$ & $43.8(11.0)$ & $42.0(7.9)$ & $42.2(8.3)$ & .188 \\
\hline Delayed recall $\mathrm{T}$ & $45.1(8.8)$ & $44.9(9.7)$ & $43.5(8.8)$ & $43.8(8.3)$ & .642 \\
\hline Working memory $\mathrm{T}$ & $49.7(8.3)$ & $47.4(8.8)$ & $46.7(9.1)$ & $46.4(7.6)$ & .066 \\
\hline Motor T & $50.5(9.9)$ & $49.9(10.4)$ & $49.3(10.0)$ & $48.1(9.6)$ & .538 \\
\hline \multicolumn{6}{|l|}{ Risk taking } \\
\hline \multicolumn{6}{|l|}{ Balloon Analog Risk Task } \\
\hline Adjusted average pumps & $28.7(14.9)$ & $29.3(12.9)$ & $29.9(14.8)$ & $30.9(12.9)$ & .826 \\
\hline Total explosions & $6[3,10]$ & $6[4,9]$ & $6[3,9]$ & $7[4,10]$ & .853 \\
\hline HIV transmission risk behaviors $\mathrm{T}^{\mathrm{c}}$ & $51.8(11.7)$ & $53.6(12.9)$ & $64.8(14.5)$ & $64.2(12.8)$ & $<.001$ \\
\hline
\end{tabular}

Values are presented as mean (SD), median [IQR], or $n(\%) . p$ values represent omnibus HIV/MDD group effects on neurobehavioral outcomes. For significant omnibus effects $(p<.05)$, pairwise comparisons were conducted and reported differences are significant at $p<.05$

${ }^{a}$ Pairwise comparisons indicated significantly higher processing speed T-scores in HIV - /MDD - compared to HIV +/MDD - and HIV + / $\mathrm{MDD}+$

${ }^{b}$ Pairwise comparisons indicated significantly higher executive functioning T-scores in HIV -/MDD - compared to HIV - /MDD +, HIV + / $\mathrm{MDD}-$, and HIV +/MDD +

${ }^{\mathrm{c}}$ Pairwise comparisons indicated significantly higher HIV transmission risk behavior T-scores in HIV +/MDD - and HIV +/MDD + compared to HIV - /MDD - and HIV - /MDD +

with HIV -/MDD - participants exhibiting higher processing speed T-scores compared to HIV +/MDD $-(d=0.45$, $p=0.005)$ and $\mathrm{HIV}+/ \mathrm{MDD}+(d=0.42, p=0.013)$ as well as higher executive functioning $\mathrm{T}$-scores compared to all three risk groups (vs. HIV $-/ \mathrm{MDD}+: d=0.45, p=0.022$; vs. HIV +/MDD - : $d=0.43, p=0.007$; vs. HIV +/MDD + : $d=0.51, p=0.003$ ).

In the full study sample, the average number of BART pumps on trials without explosions (i.e., adjusted pumps) was $29.6(\mathrm{SD}=14.1)$ and the median number of explosions was 6 (IQR: 4 to 10). HIV/MDD groups did not significantly differ with respect to average/median adjusted pumps ( $p=0.826)$ or total explosions $(p=0.853)$. On the HIV transmission risk behaviors composite T-score, HIV + participants reported higher HIV transmission risk behaviors than HIV - participants (Cohen's $d=0.95, p<0.001$ ), regardless of MDD status. MDD groups, however, did not univariably differ on HIV transmission risk behaviors within either HIV stratum $(p s>0.449)$.

\section{Interactive effects of MDD and BART on NCI}

Across the full study sample, univariable logistic regression analysis indicated a significant linear association between BART adjusted pumps and lower odds of NCI (for 1 SDunit increase: $\mathrm{OR}=0.64, p=0.002$ ). Stepwise, multivariable logistic regression models stratified by HIV serostatus examined whether MDD moderated the relationship between BART adjusted pumps and odds of NCI. Table 3 presents the results of these stepwise models. In the HIV- stratum, the overall AIC-guided step 1 model was significant $\left(\chi^{2}=10.44\right.$, Nagelkerke pseudo- $\left.R^{2}=0.12, p=0.005\right)$. With respect to retained covariates, higher sensation seeking behavior T-scores related to lower odds of NCI and a lifetime alcohol use disorder diagnosis related to higher odds of NCI. Step 2 entered MDD status and linear and quadratic adjusted pumps terms as predictors of NCI. After removing the nonsignificant quadratic adjusted pumps term $(p=0.812)$, the overall model fit of step 2 was marginally improved from step $1\left(\chi^{2}=15.72\right.$, Nagelkerke pseudo- $R^{2}=0.17, p=0.003$, log-likelihood change $p=0.071$ ) and indicated that neither MDD nor the linear effect of adjusted pumps significantly contributed to the probability of NCI above and beyond the covariates retained in step 1. Similarly, the interaction between MDD and the linear effect of adjusted pumps was not significant $(p=0.758)$ in step 3 and did not improve overall model fit $\left(\chi^{2}=15.77\right.$, Nagelkerke pseudo- $R^{2}=0.17$, $p=0.008, \log$-likelihood change $p=0.757$ ).

For the HIV + stratum, the overall fit of the AIC-guided step 1 model was significant $\left(\chi^{2}=14.03\right.$, Nagelkerke pseudo$R^{2}=0.15, p=0.003$ ). With respect to retained covariates, higher sensation seeking behavior T-scores related to lower 
Table 3 Step-wise logistic regression models examining the interactive effects of MDD and BART pumps on odds of NCI by HIV serostatus

\begin{tabular}{|c|c|c|c|c|c|c|c|c|c|}
\hline \multirow{2}{*}{$\begin{array}{l}\text { Group: HIV - } \\
\text { Parameter }\end{array}$} & \multicolumn{3}{|l|}{ Step 1} & \multicolumn{3}{|l|}{ Step 2} & \multicolumn{3}{|l|}{ Step 3} \\
\hline & $\beta(\mathrm{SE})$ & OR & $p$ & $\beta(\mathrm{SE})$ & OR & $p$ & $\beta(\mathrm{SE})$ & OR & $p$ \\
\hline \multicolumn{10}{|l|}{ Covariates } \\
\hline Lifetime AUD & $1.20(0.48)$ & 3.32 & .012 & $1.07(0.50)$ & 2.92 & .030 & $1.06(0.49)$ & 2.90 & .031 \\
\hline Sensation-seeking $\mathrm{T}$ & $-0.56(0.25)$ & 0.54 & .011 & $-0.56(0.26)$ & 0.57 & .029 & $-0.56(0.26)$ & 0.57 & .030 \\
\hline \multicolumn{10}{|l|}{ Independent effects } \\
\hline MDD & & & & $0.68(0.46)$ & 1.97 & .144 & $0.66(0.47)$ & 1.93 & .165 \\
\hline Pumps & & & & $-0.44(0.24)$ & 0.64 & .070 & $-0.39(0.29)$ & 0.68 & .187 \\
\hline \multicolumn{10}{|l|}{ Interaction effect } \\
\hline MDD $\times$ pumps & & & & & & & $-0.16(0.52)$ & 0.85 & .758 \\
\hline \multicolumn{10}{|l|}{ Model fit } \\
\hline Pseudo- $R^{2}$ & 0.12 & & .005 & 0.17 & & .003 & 0.17 & & .008 \\
\hline Log-likelihood & -65.36 & & & -62.72 & & & -62.67 & & \\
\hline Log-likelihood change & & & & 2.54 & & .071 & 0.05 & & .757 \\
\hline Group: HIV + & Step 1 & & & Step 2 & & & Step 3 & & \\
\hline Parameter & $\boldsymbol{\beta}(\mathrm{SE})$ & OR & $p$ & $\boldsymbol{\beta}(\mathrm{SE})$ & OR & $p$ & $\boldsymbol{\beta}(\mathrm{SE})$ & OR & $p$ \\
\hline \multicolumn{10}{|l|}{ Covariates } \\
\hline METH use disorder & $0.97(0.44)$ & 2.63 & .027 & $0.85(0.46)$ & 2.34 & .065 & $1.03(0.48)$ & 2.80 & .033 \\
\hline AIDS diagnosis & $0.78(0.40)$ & 2.18 & .054 & $0.73(0.42)$ & 2.07 & .084 & $0.65(0.43)$ & 1.92 & .134 \\
\hline Sensation-seeking $\mathrm{T}$ & $-0.53(0.24)$ & 0.59 & .027 & $-0.53(0.25)$ & 0.59 & .035 & $-0.55(0.26)$ & 0.58 & .036 \\
\hline \multicolumn{10}{|l|}{ Independent effects } \\
\hline MDD & & & & $0.35(0.42)$ & 1.42 & .405 & $-0.61(0.58)$ & 0.54 & .290 \\
\hline Pumps & & & & $-0.41(0.22)$ & 0.66 & .062 & $-0.28(0.27)$ & 0.75 & .283 \\
\hline Pumps $^{2}$ & & & & $0.38(0.17)$ & 1.46 & .026 & $0.11(0.20)$ & 1.12 & .575 \\
\hline \multicolumn{10}{|l|}{ Interaction effects } \\
\hline MDD × pumps & & & & & & & $-0.60(0.59)$ & 0.55 & .308 \\
\hline MDD $\times$ pumps $^{2}$ & & & & & & & $1.20(0.51)$ & 3.32 & .019 \\
\hline \multicolumn{10}{|l|}{ Model fit } \\
\hline Pseudo- $R^{2}$ & 0.15 & & .003 & 0.22 & & .002 & 0.28 & $<0.001$ & \\
\hline Log-likelihood & -74.66 & & & -70.95 & & & -68.43 & & \\
\hline Log-likelihood change & & & & 3.71 & & .059 & 2.52 & & .014 \\
\hline
\end{tabular}

Covariates in step 1 were selected using backward selection guided by Akaike's information criterion. The optimal step 1 model was based on which combination of covariates yielded the lowest overall model AIC value. Logits $(\beta)$ and odds ratio (OR) estimates for continuous independent variables reflect the effect on NCI for a 1 standard deviation change in the variable (sensation-seeking T: 10-unit change; pumps: 14-unit change). The quadratic pumps term did not reach statistical significance in the HIV- stratum model $(p=.812)$ and was therefore removed in order to accurately estimate the linear pumps term. In the HIV - stratum, eight variables were considered as covariates in step 1 because they demonstrated at least a trend-level association $(p<.10)$ with MDD (less education, METH use disorder, lifetime non-METH substance use disorder), BART adjusted pumps (younger age) or NCI (male sex, lower estimated premorbid verbal IQ, lower sensation-seeking behaviors, METH use disorder, lifetime alcohol use disorder). For the HIV + stratum, seven variables were considered as covariates because they demonstrated at least a trend-level association $(p<.10)$ with BART adjusted pumps (male sex, higher estimated premorbid verbal IQ, higher sensation-seeking behaviors, lifetime non-METH substance use disorder, absence of AIDS) or NCI (older age, lower premorbid estimated verbal IQ, lower sensationseeking behaviors, METH use disorder, AIDS diagnosis, lower nadir CD4 count). No potential covariates related to MDD status

$A U D$ alcohol use disorder, $M D D$ major depressive disorder, $M E T H$ methamphetamine

odds of NCI and diagnoses of AIDS and METH use disorder related to higher odds of NCI. Step 2 entered MDD status and linear and quadratic adjusted pumps terms as predictors of NCI. The overall model fit of step 2 was marginally improved from step $1\left(\chi^{2}=21.46\right.$, Nagelkerke pseudo$R^{2}=0.22, p=0.002$, log-likelihood change $\left.p=0.059\right)$ and indicated a significant quadratic effect of adjusted pumps $(\mathrm{OR}=1.46, p=0.026)$, but no independent main effect of MDD (OR $=1.42, p=0.405)$ on odds of NCI. In step 3 , the interaction between MDD and the quadratic effect of adjusted pumps significantly related to odds of NCI $(\mathrm{OR}=3.32, p=0.019)$ and resulted in a significant improvement in overall model fit $\left(\chi^{2}=28.63\right.$, Nagelkerke pseudo$R^{2}=0.28, p<0.001$, log-likelihood change $\left.p=0.014\right)$. In the HIV +/MDD + group, the quadratic effect of adjusted pumps on odds of NCI ( $\mathrm{OR}=3.72, p=0.005)$ exhibited a 


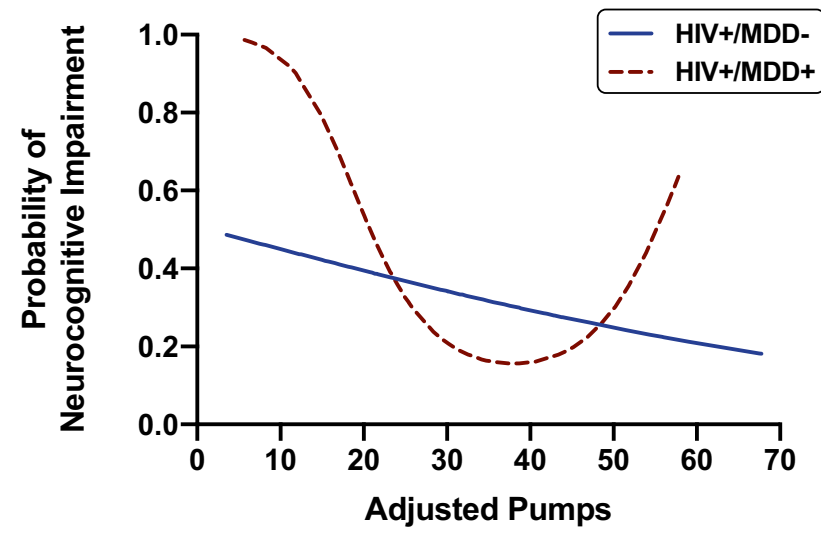

Fig. 1 Low and high risk-taking on the BART increase probability of neurocognitive impairment in HIV +/MDD + individuals but not $\mathrm{HIV}+/ \mathrm{MDD}-$

"U-shaped" pattern (Fig. 1) such that the probability of NCI was highest at the lower end of the adjusted pumps range but also elevated at the high end of the adjusted pumps range compared to intermediate levels. Conversely, there were no significant quadratic $(p=0.575)$ or linear effects $(p=0.361)$ of adjusted pumps on NCI in the HIV +/MDD - group.

\section{BART pumps and neurocognitive domains in HIV + I MDD+}

To determine which specific neurocognitive domains were driving the observed association between BART adjusted pumps and global NCI in the $\mathrm{HIV}+/ \mathrm{MDD}+$ group, we conducted separate linear regression models with adjusted pumps (linear and quadratic) as the independent variable and neurocognitive domain T-scores as the outcome variables. The combination of linear and quadratic terms for adjusted pumps accounted for a significant amount of variance in learning (linear: beta $=0.19, p<0.001$; quadratic: beta $\left.=-0.02, p<0.001 ; R^{2}=0.30\right)$ and delayed recall T-scores (linear: beta $=0.27, p=0.019$; quadratic: beta $\left.=-0.02, p<0.001 ; R^{2}=0.24\right)$. Similar to the pattern observed for NCI, learning and delayed recall were poorest at the lower end of the adjusted pumps range but also poorer at the high end of the adjusted pumps range compared to intermediate levels (Fig. 2). We did not detect significant quadratic associations for the other domains; however, the linear effect of adjusted pumps explained a significant amount of variance in executive functioning (beta $=0.23$, $p=0.010 ; R^{2}=0.12$ ) and processing speed T-scores (beta $=0.23, p=0.005 ; R^{2}=0.14$ ), with higher adjusted pumps relating to higher T-scores. The quadratic effect of adjusted pumps on learning and delayed recall and the linear effect of adjusted pumps on executive functioning and processing speed remained significant after FDR-correction
( $p$ s < 0.05). Associations between adjusted pumps and other domains (i.e., verbal fluency, working memory, and motor) did not reach statistical significance.

\section{BART pumps and HIV transmission risk behaviors}

To examine the ecological relevance of the BART, we examined linear and quadratic associations between BART adjusted pumps and self-reported risky sexual behaviors across the entire study sample and within the four study groups. Higher adjusted pumps was linearly associated with higher HIV transmission risk behaviors T-scores in both $\mathrm{MDD}+$ groups $(\mathrm{HIV}-/ \mathrm{MDD}+$ : beta $=0.48$, $p=0.005, R^{2}=0.23 ; \mathrm{HIV}+/ \mathrm{MDD}+:$ beta $=0.28$, $p=0.046, R^{2}=0.08$ ), but did not relate to HIV transmission risk behaviors T-scores in the full sample (beta $=0.10$, $\left.p=0.113, R^{2}=0.01\right)$ or within either MDD-group (HIV -/ MDD - : beta $=0.04, p=0.660, R^{2}=0.00 ; \mathrm{HIV}+/ \mathrm{MDD}-$ : beta $\left.=-0.08, p=0.515, R^{2}=0.01\right)$. Quadratic associations between adjusted pumps and HIV transmission risk behaviors T-scores did not reach statistical significance in the full sample or within any of the study groups ( $p s>0.08)$. Given the differential pattern of results (Fig. 3), we formally tested for the interaction between MDD and the linear effect of adjusted pumps in the entire sample, controlling for HIV serostatus and METH use disorder. This model indicated a significant interaction effect between MDD and adjusted pumps (beta $=0.38, p=0.002$ ) on HIV transmission risk behaviors in the presence of independent effects of HIV serostatus (beta $=12.34, p<0.001)$ and METH use disorder (beta $=8.23, p<0.001)$.

\section{Secondary analyses: methamphetamine use}

Although statistical covariation for METH use did not attenuate the significant associations of BART performance with NCI and HIV transmission risk behaviors, METH use has known adverse effects on neurobehavior and may moderate the relationships between our primary study variables. Thus, a series of post hoc analyses probed the moderating effects of METH use on significant associations established in the primary study analyses. Similar to the lack of HIV/ MDD group differences in BART performance, METH use disorder did not significantly relate to BART adjusted pumps in the full sample (METH - mean $[\mathrm{SD}]=29.4$ [13.6] vs. METH + mean $[\mathrm{SD}]=29.9$ [14.8], $d=0.03, p=0.804$ ) or within each HIV/MDD group ( $p s>0.426$ ). METH use disorder did not significantly interact with the quadratic effect of BART adjusted pumps on NCI in the HIV + /MDD + group $(\mathrm{OR}=1.43, p=0.755)$, and the quadratic effect of adjusted pumps on NCI in the HIV +/MDD + group persisted in sensitivity analyses stratified by $\mathrm{METH}-(n=30 ; \mathrm{OR}=3.73$, $p=0.037)$ and $\mathrm{METH}+(n=26 ; \mathrm{OR}=4.90, p=0.097)$. 
A Learning

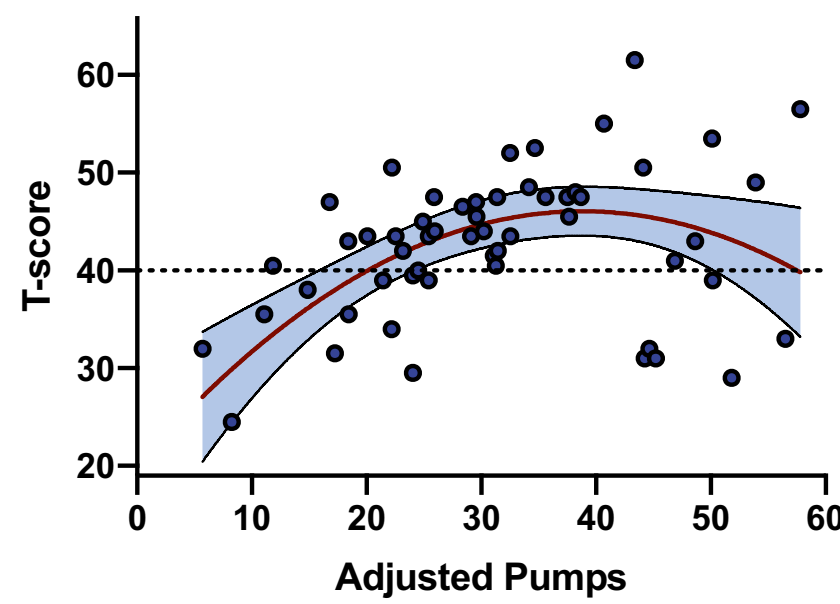

C

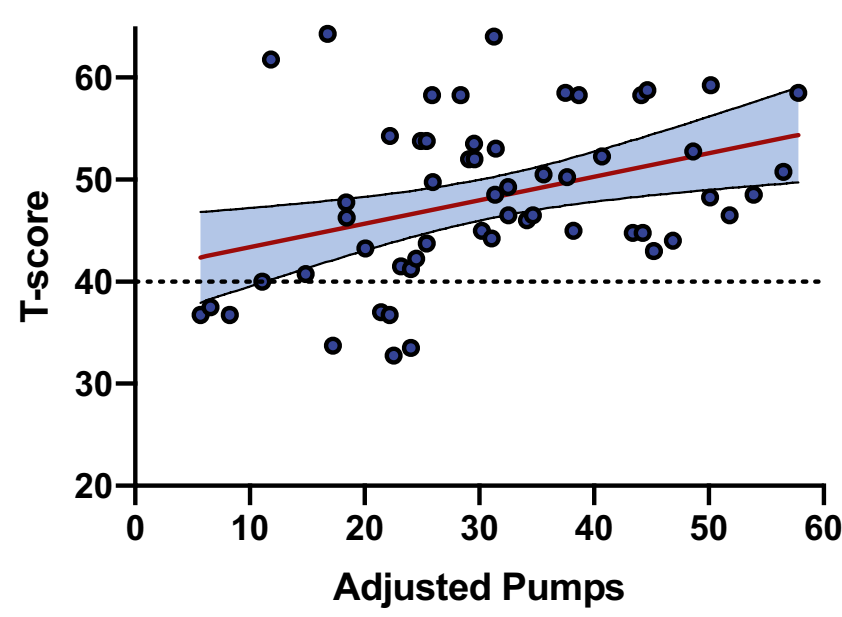

B Delayed Recall

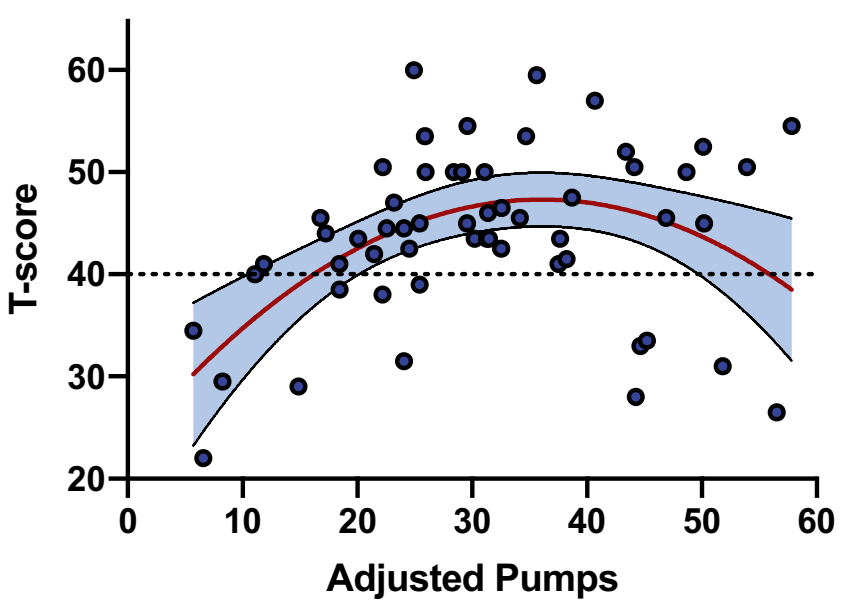

D Executive Function

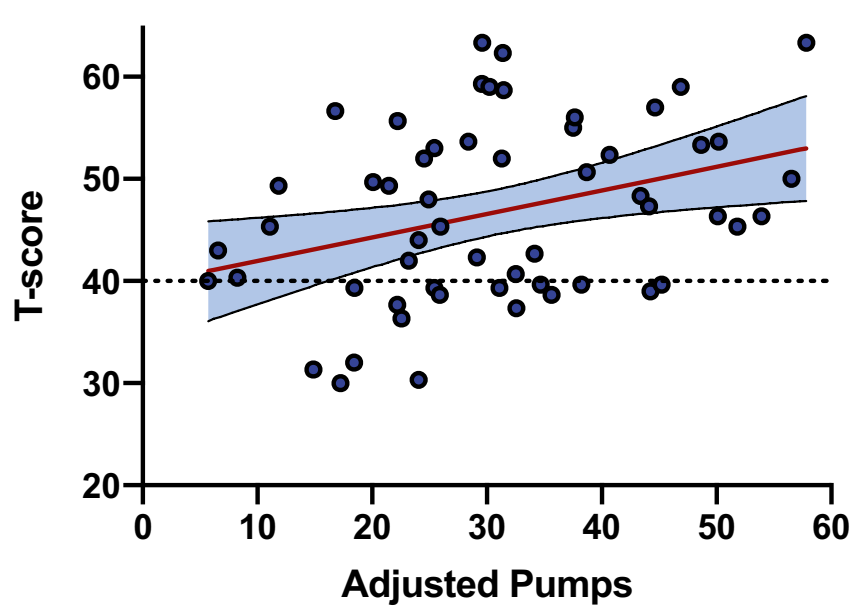

Fig. 2 Linear and quadratic effects of risk-taking on domain-specific neurocognition in HIV +/MDD +

Similarly, METH use disorder did not further moderate the MDD x BART adjusted pumps interaction on HIV

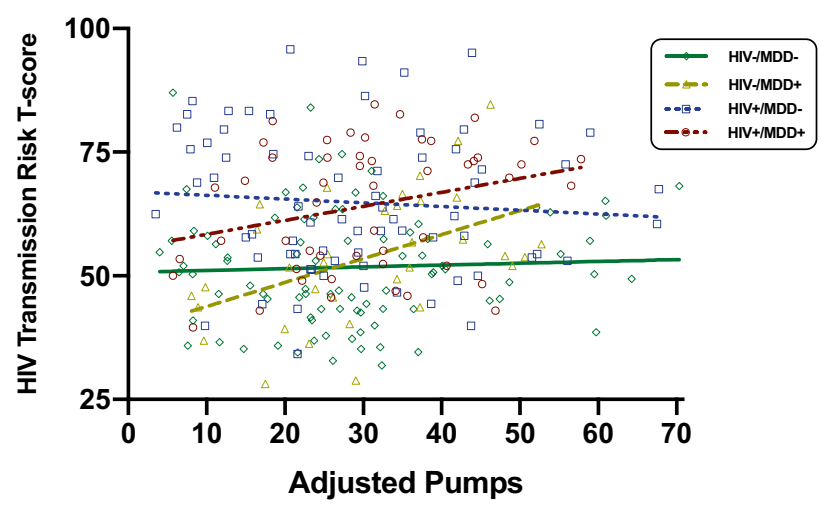

Fig. 3 Higher risk-taking on the BART is associated with higher HIV transmission risk behaviors only in MDD + individuals transmission risk behaviors $\mathrm{T}$-scores in the full sample (beta $=0.19, p=0.446$ ), and the MDD $\times$ BART adjusted pumps interaction persisted in sensitivity analyses stratified by METH $-(n=140$; beta $=0.29, p=0.073)$ and $\operatorname{METH}+(n=102 ;$ beta $=0.24, p=0.011)$.

\section{Discussion}

The present study adds to the literature on risk-taking in HIV by characterizing the concordance of NCI and risktaking propensity, as indexed by BART adjusted pumps, in a cohort of individuals stratified by HIV serostatus and MDD. Neurocognition was strongest in the HIV -/MDD - group, particularly compared to the HIV + groups, whereas BART adjusted pumps did not significantly differ across the four study groups. Initial observation of the data across the entire 
study sample suggested that those with NCI exhibited fewer BART adjusted pumps than those without NCI. However, after stratifying by HIV serostatus and examining curvilinear BART effects on NCI, a quadratic pattern emerged in the HIV + group such that rates of NCI were elevated among PWH whose average adjusted pumps fell in the lower end ("risk-averse") or the higher end ("risk-taking") of the BART adjusted pumps range compared to intermediate levels. Furthermore, interaction effects indicated that this quadratic pattern of risk-taking propensity and NCI was unique to the dual-risk HIV +/MDD + group and predicted NCI above and beyond comorbid METH use disorder, a history of AIDS, and sensation-seeking traits. Conversely, BART adjusted pumps only exhibited a trend-level linear association with NCI in HIV- individuals that was not moderated by MDD status. Overall, these findings further support a role for affect in modulating HIV-related neurobehavioral functioning and suggest that low and high risk-taking phenotypes are both indicators of neurocognitive dysfunction when lifetime syndromic depression is superimposed upon HIV disease.

Our stepwise regression models indicated a notably large moderation effect of depression on the association between the BART and NCI in PWH. The pseudo- $R^{2}$ effect size estimate almost doubled from the step 1 model with only covariates (pseudo- $R^{2}=0.15$ ) to the step 3 model that included the MDD interaction with linear and quadratic adjusted BART pumps (pseudo- $R^{2}=0.28$ ). Moreover, the quadratic BART effect indicated large alterations to the BART and NCI relationship as a function of BART pumps and $24-30 \%$ of the variance in learning and delayed recall was explained by the quadratic BART effect. Neurocognitive domain-specific analyses suggested neurocognitive profiles differed across the spectrum of risk-taking propensity in the HIV +/MDD + group. Individuals with average adjusted pumps on the BART that fell within the lower range had the highest probability of NCI and accordingly exhibited diffuse decrements across the domains of processing speed, executive function, learning, and memory. Strenziok et al. (2011) similarly reported that patients with behavioral variant frontotemporal dementia exhibited fewer BART adjusted pumps compared to controls and that impaired stimulus-reinforcement learning across the task related to greater atrophy in the right lateral orbitofrontal cortex (Strenziok et al. 2011), a region directly implicated in the pathogenesis of depression (Feffer et al. 2018; Yu et al. 2018). HIV +/MDD + individuals with high BART adjusted pumps also exhibited an elevated risk for NCI, yet this risk was driven primarily by poor learning and memory in the context of better processing speed and executive function.

The dissociation between poor learning/memory and intact executive function/processing speed in the high risk-taking HIV + /MDD + group is particularly notable when considering that the BART exhibited a positive, linear association with HIV transmission risk behaviors in this group. This converges with two prior studies that reported better IGT performance related to greater risky behaviors (i.e., sexual risk-taking, substance use) in PWH with high levels of emotional distress or sensation seeking personality traits (Golub et al. 2016; Wardle et al. 2010). Several component processes are involved in risky decision-making, including the valuation of potential outcomes, perceived likelihood of outcomes, motivation to pursue positive outcomes, tolerance for negative outcomes, and capacity to learn from past outcomes (Banich and Floresco 2019; Orsini et al. 2019). These data in conjunction with our findings suggest that certain aspects of the cognitive circuitry underlying risky decision-making, such as efficiently processing information to assign values to potential outcomes and subsequently formulating a plan to pursue high-reward albeit risky outcomes, must be intact in order for emotional factors to influence the execution of risky behaviors. In contrast, the poor learning and memory in the HIV + I MDD + group may be less critical for the execution of a risky behavior and may rather reflect an impaired capacity to learn from past negative outcomes, thereby also contributing to risk-taking propensity.

Although performance on behavioral risk tasks may reflect acquired neurocognitive deficits that underpin the component processes of decision-making, the somatic marker hypothesis describes the strong influence of affective states on risky decision-making (Bechara et al. 2000; Lerner et al. 2015). This hypothesis postulates that when confronted with a decision, individuals receive bioregulatory signals (i.e., somatic markers) rich in affective information regarding possible response outcomes (Bechara et al. 2000; Damasio 1996). Under this somatic marker framework, suboptimal decision-making may manifest from the failure to integrate these somatic markers with the cognitive components of decision-making, particularly in the setting of monoaminergic dysfunction (Bechara et al. 2000; Rogers et al. 1999). For example, depressed individuals may ineffectively process affective signals during decision-making due to altered reward and/or punishment sensitivity arising from disrupted cortico-striatal reward signaling (Eshel and Roiser 2010; Husain and Roiser 2018; Must et al. 2006). Importantly, this may help explain the heterogeneity in risk-taking and neurocognitive performance observed in our historically-depressed group of PWH, as altered reward processing may generate affective signals that contribute to both risk averse (Hevey et al. 2017; Smoski et al. 2008) and risk-taking behaviors (Whittle et al. 2015), particularly in PWH (Cook et al. 2015).

The relationship between BART performance and $\mathrm{NCI}$ in individuals without HIV was weaker than our observations 
in PWH and was not moderated by lifetime MDD status. From a statistical perspective, the specificity of our findings to PWH may be partially explained by higher rates of $\mathrm{NCI}$ in HIV + /MDD - and HIV +/MDD + compared to the HIV - / MDD - group, which comprised $72 \%$ of the HIV - sample. Furthermore, the HIV -/MDD + reported similar levels of current depressive symptoms to the HIV +/MDD- group and substantially milder symptoms compared to the HIV + I MDD + group. The higher levels of current neurocognitive and affective dysfunction seen in the HIV +/MDD + sample likely enhanced power to detect MDD-dependent associations between the BART and neurocognition, which would have been less likely had groups been classified based on current MDD criteria given the small sample size. Nevertheless, BART adjusted pumps explained $23 \%$ of the variance in risky sexual behaviors in the HIV-/MDD + group, underscoring the convergent validity of the BART with real-world risk behaviors in individuals with histories of clinical depression.

Although partially explained by the above mentioned findings, the lack of differences across groups on BART performance may be explained by additional factors, and is worth discussion, particularly given inconsistencies in the literature. For example, some studies have reported that PWH, particularly those with elevated affective distress, exhibit greater risk-taking across several behavioral risk tasks (Hardy et al. 2006; Martin et al. 2016; Paydary et al. 2016; Thames et al. 2012). Others have failed to identify main effects of HIV serostatus on risky decision-making, particularly the IGT, yet have detected HIV-related influences on risk-taking that are conditional on neurocognitive and affective factors (Gonzalez et al. 2005; Iudicello et al. 2013). This is consistent with our observations and highlights the importance of understanding the affective and neurocognitive conditions that contextualize BART performance, as examination of BART performance in isolation did not adequately discriminate HIV-related and MDDrelated neurobehavioral dysfunction. There are other potential explanations for the lack of group differences across BART performance. Specific to our study, our comparison groups (e.g., HIV - , MDD -) were drawn from the same parent study as our groups of interest (TMARC), which overall had high proportions of substance use (e.g., METH, alcohol) use disorders and elevated behavioral characteristics (e.g., impulsivity, sensation seeking) that have been strongly associated with risk taking propensity on the BART (Kohno et al. 2014; Lejuez et al. 2002). The lack of group differences may also be attributed to the BART outcome measure used (e.g., average adjusted number of pumps), which while extensively used in the literature, may not fully capture the intricacies of risk-taking propensity in these populations. Future research exploring other metrics, such as those aimed at elucidating the profile of performance on the BART (e.g., inter-trial variability or differences in behavior following balloon explosions versus rewards; Canning et al. 2021), could provide valuable insight into risky decisionmaking in these populations, as would the development of computational models that tap into multiple facets of risktaking propensity (Park et al. 2021; Wallsten et al. 2005).

Growing evidence indicates that a subset of clinically depressed patients display an enhanced inflammatory state that is linked to monoaminergic dysregulation in corticostriatal circuits underpinning motivation and reward (Felger et al. 2016; Felger and Miller 2012; Haroon et al. 2018). Consistent with this immunophenotype of depression, we have recently characterized associations between HIV-associated neuroinflammation, depressive symptoms, and CSF dopaminergic deficits (Ellis et al. 2020; Saloner et al. 2020a). Moreover, chronic depression predicts steeper declines in executive function and recall and moderates the acute effects of systemic inflammation on psychomotor and cognitive slowing in PWH (Paolillo et al. 2020; Saloner et al. 2020a). Risk-taking profiles may show high concordance with neurocognitive dysfunction in depressed PWH given that HIV-associated neuroinflammation preferentially targets these frontostriatal circuits that support higher-order neurocognitive functions, emotional regulation, and reward processing (Soontornniyomkij et al. 2016; Woods et al. 2009). A recent study in stimulant-using PWH identified an association between higher BART scores and greater tryptophan degradation (Lee et al. 2020), a marker of immune activation and serotonin deficiency that is implicated in HIV-related depression (Gostner et al. 2015). At the neurocircuit level, HIV disease has been associated with reduced resting-state frontostriatal connectivity, which in turn correlated with higher odds of NCI (Ipser et al. 2015). PWH also exhibit greater activation of the prefrontal cortex during a monetary decision-making task, suggestive of decreased neural efficiency, and this compensatory neural activation of the prefrontal cortex is related to higher BART scores and lower nadir CD4 counts (Meade et al. 2016).

This study is among the first to model linear and quadratic patterns of risk-taking propensity, measured with the BART, as it relates to neurocognition and real-world risk-taking in a wellcharacterized cohort of individuals stratified by HIV serostatus and MDD. It is worth noting several limitations to the present study. Due to the overarching parent study (TMARC) aims (Saloner et al. 2020b; Soontornniyomkij et al. 2016), a significant proportion of participants had METH use disorder diagnoses, including the HIV- and MDD- samples. METH use is associated with risk-taking behaviors (Gonzalez et al. 2007) and increases risk for NCI in PWH (Carey et al. 2004; Rippeth et al. 2004). Consistently, METH use was associated with NCI in our study, but only in the HIV + group. However, our main study findings, namely, the quadratic BART association with NCI in the HIV +/MDD + group, persisted while controlling for METH use as a covariate. These findings were 
further strengthened by sensitivity analyses demonstrating that the quadratic BART association with $\mathrm{NCI}$ in the HIV + / $\mathrm{MDD}+$ group persisted in both METH - and METH + individuals. To provide further support for our findings in light of the high prevalence of METH use in our sample, we conducted post hoc analyses to probe the potential influence of METH use on risk-taking propensity and potential interactive effects with significant associations established in the primary study analyses. Similar to the lack of HIV/MDD group differences in BART performance, METH use disorder did not significantly relate to BART adjusted pumps in the full sample or within each HIV/MDD group. Moreover, METH use disorder did not significantly interact with the quadratic effect of BART adjusted pumps on NCI in the HIV +/MDD + group. Lastly, neither duration (i.e., lifetime total days of METH use) and amount (lifetime total grams consumed) related to probability of NCI or BART adjusted pumps, regardless of HIV or MDD status (data not shown). While METH use diagnosis and characteristics did not appear to moderate our primary findings, it is nonetheless essential to consider in future studies examining risk taking propensity or profiles of risk behavior, particularly in HIV and other populations in which use is highly prevalent. Other factors such as personality traits associated with METH use (e.g., reward sensitivity) may also play a role in risk propensity (e.g., White et al. 2007) and should be carefully considered in future research.

Second, our data importantly integrates an experimental cognitive task (i.e., BART) standard clinical neuropsychological tests, and ecologically relevant risky sexual behaviors, yet these neurobehavioral data do not directly measure the frontal systems neurocircuitry implicated in HIV and depression. Future studies that incorporate biological units of analysis, such as structural/functional neuroimaging and inflammatory biomarkers, would allow us to formally examine the putative neurobiological mechanisms underpinning risk-taking behavior in this population. Next, the cross-sectional design of our study does not allow us to confirm the directionality or stability of our findings. We intentionally entered BART performance as a predictor of neurocognition in order to examine quadratic patterns of risk-taking propensity; however, it is also reasonable to conceptualize neurocognition as a determinant of risky decision-making and therefore our independent and dependent variables are not discussed in terms of causality. Our study population of PWH was also predominantly men, consistent with the demographics of PWH in San Diego, with a high prevalence of comorbid substance use disorders. Prior studies have described sex differences in IGT performance and NCI in PWH (Martin et al. 2016; Rubin et al. 2019; Sundermann et al. 2018) as well as sex influences on BART performance in HIV-seronegative adults (Lejuez et al. 2002; Lighthall et al. 2009; White et al. 2007), and future work should therefore aim to elucidate sex differences in the neurocognitive correlates of BART performance in PWH.
Last, our overall study sample likely reflects a group high in characteristics associated with risk-taking, higher baseline levels of risk-taking, NCI, and emotional dysregulation than a sample without a history of addictive behaviors. The common co-occurrence of substance use disorders in PWH with and without MDD enhances the ecological validity of our findings, however future studies focused on MDD in people without significant substance disorders should help to validate the specificity of the relationships that we report. In addition to risky sexual behaviors, future analyses should examine the moderating influence of lifetime and current MDD on the relationship between BART performance and other facets of real world risk-taking such as substance use behaviors in PWH.

Our findings challenge prior studies that have assumed a linear relationship between risk-taking propensity and neurocognition, particularly in groups with underlying frontostriatal dysfunction. Moreover, these findings advocate for neurobehavioral screenings that incorporate both affective and neurocognitive measures in PWH. Given the large influence of depression on the relationship between risk-taking and neurocognition, clinicians may consider further screening for risky behaviors in PWH who present with comorbid depression histories and intact executive functioning/ processing speed but impaired learning/memory. Interventions aimed at ameliorating depressive symptoms and/or enhancing sensitivity to the adverse consequences of risktaking may help reduce HIV transmission risk in these individuals. Conversely, the absence of risky decision-making in depressed PWH may not purely reflect low risk-taking propensity, but may also reflect overlapping neurocognitive and depressive symptoms (e.g., executive dysfunction and apathy) that contribute to impaired decision-making. Thus, diffusely impaired and "deactivated" individuals should also be monitored in the event that they experience "activations" in aspects of their neurocognitive and affective profile that facilitate the execution of risky behaviors.

Acknowledgments *The Translational Methamphetamine AIDS Research Center (TMARC) is supported by Center award P50DA026306 from the National Institute on Drug Abuse (NIDA) and is affiliated with the University of California, San Diego (UCSD), the SanfordBurnham Medical Discovery Institute (SBMDI), and the University of California, Irvine (UCI). The TMARC comprises: Administrative Coordinating Core (ACC)-Executive Unit: Director-Igor Grant, M.D.; Co-Directors-Ronald J. Ellis, M.D., Ph.D., Scott L. Letendre, M.D., and Cristian L. Achim, M.D., Ph.D.; Center Manager-Mariana Cherner, Ph.D.; Associate Center Managers-Erin E. Morgan, Ph.D. and Jared Young, Ph.D.; Data Management and Information Systems (DMIS) Unit: Ian S. Abramson, Ph.D. (Unit Chief), Clint Cushman, B.A. (Unit Manager); ACC - Statistics Unit: Florin Vaida, Ph.D. (Unit Chief), Ian S. Abramson, Ph.D., Reena Deutsch, Ph.D., Anya Umlauf, M.S.; ACC-Participant Unit: J. Hampton Atkinson, M.D. (Unit Chief), Jennifer Marquie-Beck, M.P.H. (Unit Manager); Behavioral Assessment and Medical (BAM) Core-Neuromedical and Laboratory 
Unit (NLU): Scott L. Letendre, M.D. (Core Co-Director/NLU Chief), Ronald J. Ellis, M.D., Ph.D.; BAM Core-Neuropsychiatric Unit (NPU): Robert K. Heaton, Ph.D. (Core Co-Director/NPU Chief), J. Hampton Atkinson, M.D., Thomas D. Marcotte, Ph.D., Erin E. Morgan, Ph.D., Matthew Dawson (NPU Manager); Neuroimaging (NI) Core: Gregory G. Brown, Ph.D. (Core Director), Thomas T. Liu, Ph.D., Miriam Scadeng, Ph.D., Christine Fennema-Notestine, Ph.D., Sarah L. Archibald, M.A., John R. Hesselink, M.D., Mary Jane Meloy, Ph.D., Craig E.L. Stark, Ph.D.; Neuroscience and Animal Models (NAM) Core: Cristian L. Achim, M.D., Ph.D. (Core Director), Marcus Kaul, Ph.D., Virawudh Soontornniyomkij, M.D.; Pilot and Developmental (PAD) Core: Mariana Cherner, Ph.D. (Core Director), Stuart A. Lipton, M.D., Ph.D.; Project 1: Arpi Minassian, Ph.D. (Project Director), William Perry, Ph.D., Mark A. Geyer, Ph.D., Jared W. Young, Ph.D.; Project 2: Amanda B. Grethe, Ph.D. (Project Director), Susan F. Tapert, Ph.D., Assawin Gongvatana, Ph.D.; Project 3: Erin E. Morgan, Ph.D. (Project Director), Igor Grant, M.D.; Project 4: Samuel Barnes, Ph.D. (Project Director).; Project 5: Marcus Kaul, Ph.D. (Project Director), Ana Sanchez, Ph.D.

The views expressed in this article are those of the authors and do not reflect the official policy or position of the US Government.

Funding This work was supported by awards from the National Institutes of Health: Translational Methamphetamine AIDS Research Center (TMARC)*-center award P50DA026306 from NIDA; T32DA031098 award from NIDA (stipend support to M.A.H.); K23DA037793 and R01DA047879 awards from NIDA (salary support to J.E.I.); F31AG064989 award from NIA (stipend support to R.S.).

\section{Declarations}

Conflict of interest The authors declare no competing interests.

Open Access This article is licensed under a Creative Commons Attribution 4.0 International License, which permits use, sharing, adaptation, distribution and reproduction in any medium or format, as long as you give appropriate credit to the original author(s) and the source, provide a link to the Creative Commons licence, and indicate if changes were made. The images or other third party material in this article are included in the article's Creative Commons licence, unless indicated otherwise in a credit line to the material. If material is not included in the article's Creative Commons licence and your intended use is not permitted by statutory regulation or exceeds the permitted use, you will need to obtain permission directly from the copyright holder. To view a copy of this licence, visit http://creativecommons.org/licenses/by/4.0/.

\section{References}

Akaike H (1974) A new look at the statistical model identification. IEEE Trans Autom Control 19:716-723

Anand P, Springer SA, Copenhaver MM, Altice FL (2010) Neurocognitive impairment and HIV risk factors: a reciprocal relationship. AIDS Behav 14:1213-1226

Arseniou S, Arvaniti A, Samakouri M (2014) HIV infection and depression. Psychiatry Clin Neurosci 68:96-109

Balagueró MA, Jodar Vicente M, García Molina A, Tormos JM, Roig Rovira T (2016) Balloon analogue risk task to assess decisionmaking in acquired brain injury. Int J Psychol Res (Medellin) 9:30-39

Balogh KN, Mayes LC, Potenza MN (2013) Risk-taking and decisionmaking in youth: relationships to addiction vulnerability. J Behav Addict 2. https://doi.org/10.1556/JBA.2.2013.1.1
Banich MT, Floresco S (2019) Reward systems, cognition, and emotion: introduction to the special issue. Cogn Affect Behav Neurosci 19:409-414

Bechara A (2007) Iowa gambling task. Psychological Assessment Resources Inc, Lutz, FL

Bechara A, Damasio AR, Damasio H, Anderson SW (1994) Insensitivity to future consequences following damage to human prefrontal cortex. Cognition 50:7-15

Bechara A, Damasio H, Damasio AR (2000) Emotion, decision making and the orbitofrontal cortex. Cereb Cortex 10:295-307

Beck A, Steer R, Brown G (1996) Manual for Beck Depression Inventory II (BDI-II). Psychology Corporation, San Antonio, TX

Benjamini Y, Hochberg Y (1995) Controlling the false discovery rate: a practical and powerful approach to multiple testing. J Roy Stat Soc: Ser B (methodol) 57:289-300

Bishara AJ, Pleskac TJ, Fridberg DJ, Yechiam E, Lucas J, Busemeyer JR, Finn PR, Stout JC (2009) Similar processes despite divergent behavior in two commonly used measures of risky decision making. J Behav Decis Mak 22:435-454

Blackstone K, Moore DJ, Franklin DR, Clifford DB, Collier AC, Marra CM, Gelman BB, McArthur JC, Morgello S, Simpson DM, Ellis RJ, Atkinson JH, Grant I, Heaton RK (2012) Defining neurocognitive impairment in HIV: deficit scores versus clinical ratings. Clin Neuropsychol 26:894-908

Burnham KP, Anderson DR (2004) Multimodel inference: understanding AIC and BIC in model selection. Sociol Methods Res 33:261-304

Canning JR, Schallert MR, Larimer ME (2021) A systematic review of the Balloon Analogue Risk Task (BART) in alcohol research. Alcohol Alcohol

Carey CL, Woods SP, Gonzalez R, Conover E, Marcotte TD, Grant I, Heaton RK (2004) Predictive validity of global deficit scores in detecting neuropsychological impairment in HIV infection. J Clin Exp Neuropsychol 26:307-319

Cook SH, Valera P, Wilson PA, Network TAT, for HIV, AIDS Interventions (2015) HIV status disclosure, depressive symptoms, and sexual risk behavior among HIV-positive young men who have sex with men. J Behav Med 38:507-517

Crooks D, Tsui J, Anderson B, Dossabhoy S, Herman D, Liebschutz JM, Stein MD (2015) Differential risk factors for HIV drug and sex risk-taking among non-treatment-seeking hospitalized injection drug users. AIDS Behav 19:405-411

Cysique LA, Franklin D, Jr., Abramson I, Ellis RJ, Letendre S, Collier A, Clifford D, Gelman B, McArthur J, Morgello S, Simpson D, McCutchan JA, Grant I, Heaton RK, Group C, Group H (2011) Normative data and validation of a regression based summary score for assessing meaningful neuropsychological change. J Clin Exp Neuropsychol 33:505-522

Damasio AR (1996) The somatic marker hypothesis and the possible functions of the prefrontal cortex. Philos Trans R Soc Lond B Biol Sci 351:1413-1420

DeHart DD, Birkimer JC (1997) Trying to practice safer sex: development of the sexual risks scale. J Sex Res 34:11-25

Ellis R, Langford D, Masliah E (2007) HIV and antiretroviral therapy in the brain: neuronal injury and repair. Nat Rev Neurosci 8:33-44

Ellis RJ, Letendre SL, Atkinson JH, Clifford D, Collier AC, Gelman BB, Marra C, McCutchan JA, Morgello S, Sacktor N, Tang B, Heaton RK (2020) Higher levels of plasma inflammation biomarkers are associated with depressed mood and quality of life in aging, virally suppressed men, but not women, with HIV. Brain Behav Immun Health 100121

Eshel N, Roiser JP (2010) Reward and Punishment Processing in Depression. Biol Psychiat 68:118-124

Fecteau S, Levasseur-Moreau J, García-Molina A, Kumru H, Vergara RP, Bernabeu M, Roig T, Pascual-Leone A, Tormos JM (2013) 
Risk taking in hospitalized patients with acute and severe traumatic brain injury. PLoS One 8:e83598

Feffer K, Fettes P, Giacobbe P, Daskalakis ZJ, Blumberger DM, Downar J (2018) 1Hz rTMS of the right orbitofrontal cortex for major depression: safety, tolerability and clinical outcomes. Eur Neuropsychopharmacol 28:109-117

Felger JC, Li Z, Haroon E, Woolwine BJ, Jung MY, Hu X, Miller AH (2016) Inflammation is associated with decreased functional connectivity within corticostriatal reward circuitry in depression. Mol Psychiatry 21:1358-1365

Felger JC, Miller AH (2012) Cytokine effects on the basal ganglia and dopamine function: the subcortical source of inflammatory malaise. Front Neuroendocrinol 33:315-327

Golub SA, Thompson LI, Kowalczyk WJ (2016) Affective differences in Iowa Gambling Task performance associated with sexual risk taking and substance use among HIV-positive and HIVnegative men who have sex with men. J Clin Exp Neuropsychol 38:141-157

Gonzalez R, Bechara A, Martin EM (2007) Executive functions among individuals with methamphetamine or alcohol as drugs of choice: preliminary observations. J Clin Exp Neuropsychol 29:155-159

Gonzalez R, Vassileva J, Bechara A, Grbesic S, Sworowski L, Novak RM, Nunnally G, Martin EM (2005) The influence of executive functions, sensation seeking, and HIV serostatus on the risky sexual practices of substance-dependent individuals. J Int Neuropsychol Soc 11:121-131

Gostner JM, Becker K, Kurz K, Fuchs D (2015) Disturbed amino acid metabolism in HIV: association with neuropsychiatric symptoms. Front Psych 6:97-97

Grace J (2001) Frontal systems behavior scale: professional manual. Psychological Assessment Resources Inc, Lutz, FL

Hardy DJ, Hinkin CH, Levine AJ, Castellon SA, Lam MN (2006) Risky decision making assessed with the gambling task in adults with HIV. Neuropsychology 20:355-360

Haroon E, Chen X, Li Z, Patel T, Woolwine BJ, Hu XP, Felger JC, Miller AH (2018) Increased inflammation and brain glutamate define a subtype of depression with decreased regional homogeneity, impaired network integrity, and anhedonia. Transl Psychiatry 8:189-189

Heaton RK, Clifford DB, Franklin DR, Jr., Woods SP, Ake C, Vaida F, Ellis RJ, Letendre SL, Marcotte TD, Atkinson JH, RiveraMindt M, Vigil OR, Taylor MJ, Collier AC, Marra CM, Gelman BB, McArthur JC, Morgello S, Simpson DM, McCutchan JA, Abramson I, Gamst A, Fennema-Notestine C, Jernigan TL, Wong J, Grant I, Group C (2010) HIV-associated neurocognitive disorders persist in the era of potent antiretroviral therapy: CHARTER Study. Neurology 75:2087-2096

Heaton RK, Marcotte TD, Mindt MR, Sadek J, Moore DJ, Bentley H, McCutchan JA, Reicks C, Grant I (2004a) The impact of HIVassociated neuropsychological impairment on everyday functioning. J Int Neuropsychol Soc 10:317-331

Heaton RK, Miller SW, Taylor MJ, Grant I (2004b) Revised comprehensive norms for an expanded Halstead Reitan battery: demographically adjusted neuropsychological norms for African American and Caucasian adults. Psychological Assessment Resources Inc, Lutz, FL

Heaton RK, Taylor MJ, Manly J (2003) Demographic effects and use of demographically corrected norms with the WAIS-III and WMSIII. Clinical interpretation of the WAIS-III and WMS-III. Academic Press, San Diego, CA, US, pp 181-210

Hevey D, Thomas K, Laureano-Schelten S, Looney K, Booth R (2017) Clinical depression and punishment sensitivity on the BART. Front Psychol 8:670-670

Hult B, Chana G, Masliah E, Everall I (2008) Neurobiology of HIV. Int Rev Psychiatry 20:3-13
Hunt MK, Hopko DR, Bare R, Lejuez CW, Robinson EV (2005) Construct validity of the balloon analog risk task (BART) associations with psychopathy and impulsivity. Assessment 12:416-428

Husain M, Roiser JP (2018) Neuroscience of apathy and anhedonia: a transdiagnostic approach. Nat Rev Neurosci 19:470-484

Ipser JC, Brown GG, Bischoff-Grethe A, Connolly CG, Ellis RJ, Heaton RK, Grant I, Translational Methamphetamine ARCG (2015) HIV infection is associated with attenuated frontostriatal intrinsic connectivity: a preliminary study. J Int Neuropsychol Soc : JINS 21:203-213

Iudicello JE, Woods SP, Cattie JE, Doyle K, Grant I, Group HIVNRP (2013) Risky decision-making in HIV-associated neurocognitive disorders (HAND). Clin Neuropsychol 27:256-275

James BD, Boyle PA, Yu L, Han SD, Bennett DA (2015) Cognitive decline is associated with risk aversion and temporal discounting in older adults without dementia. PLoS ONE 10:e0121900-e0121900

Kahneman D, Tversky A (1979) Prospect theory: an analysis of decision under risk. Econometrica 47:263-291

Kalichman SC, Johnson JR, Adair V, Rompa D, Multhauf K, Kelly JA (1994) Sexual sensation seeking: Scale development and predicting AIDS-risk behavior among homosexually active men. J Pers Assess 62:385-397

Kalichman SC, Rompa D (1995) Sexual sensation seeking and sexual compulsivity scales: Validity, and predicting HIV risk behavior. J Pers Assess 65:586-601

Kohno M, Morales AM, Ghahremani DG, Hellemann G, London ED (2014) Risky decision making, prefrontal cortex, and mesocorticolimbic functional connectivity in methamphetamine dependence. JAMA Psychiat 71:812-820

Kreek MJ, Nielsen DA, Butelman ER, LaForge KS (2005) Genetic influences on impulsivity, risk taking, stress responsivity and vulnerability to drug abuse and addiction. Nat Neurosci 8:1450-1457

Lauriola M, Panno A, Levin IP, Lejuez CW (2014) Individual differences in risky decision making: a meta-analysis of sensation seeking and impulsivity with the balloon analogue risk task. J Behav Decis Mak 27:20-36

Lee J, Lee JY, Meade CS, Cohn M, Chahine A, Dilworth SE, Magidson JF, Gouse H, Fuchs D, Carrico AW (2020) Tryptophan degradation is associated with risk-taking propensity in methamphetamine users with treated HIV infection. J Neurovirol 26:779-784

Lejuez CW, Read JP, Kahler CW, Richards JB, Ramsey SE, Stuart GL, Strong DR, Brown RA (2002) Evaluation of a behavioral measure of risk taking: the Balloon Analogue Risk Task (BART). J Exp Psychol Appl 8:75

Lejuez CW, Simmons BL, Aklin WM, Daughters SB, Dvir S (2004) Risk-taking propensity and risky sexual behavior of individuals in residential substance use treatment. Addict Behav 29:1643-1647

Lerner JS, Li Y, Valdesolo P, Kassam KS (2015) Emotion and decision making. Annu Rev Psychol 66:799-823

Lighthall NR, Mather M, Gorlick MA (2009) Acute stress increases sex differences in risk seeking in the Balloon Analogue Risk Task. PLOS ONE 4:e6002

Llewellyn DJ (2008) The psychology of risk taking: toward the integration of psychometric and neuropsychological paradigms. Am J Psychol 363-376

Marquine MJ, Iudicello JE, Morgan EE, Brown GG, Letendre SL, Ellis RJ, Deutsch R, Woods SP, Grant I, Heaton RK (2014) "Frontal systems" behaviors in comorbid human immunodeficiency virus infection and methamphetamine dependency. Psychiatry Res 215:208-216

Martin E, Gonzalez R, Vassileva J, Maki PM, Bechara A, Brand M (2016) Sex and HIV serostatus differences in decision making under risk among substance-dependent individuals. J Clin Exp Neuropsychol 38:404-415 
Martin EM, DeHaan S, Vassileva J, Gonzalez R, Weller J, Bechara A (2013) Decision making among HIV+ drug using men who have sex with men: a preliminary report from the Chicago Multicenter AIDS Cohort Study. J Clin Exp Neuropsychol 35:573-583

Martin EM, Pitrak DL, Weddington W, Rains NA, Nunnally G, Nixon H, Grbesic S, Vassileva J, Bechara A (2004) Cognitive impulsivity and HIV serostatus in substance dependent males. J Int Neuropsychol Soc 10:931-938

McNair DM (1992) Profile of mood states. Educational and Industrial Testing Service

Meade CS, Cordero DM, Hobkirk AL, Metra BM, Chen NK, Huettel SA (2016) Compensatory activation in fronto-parietal cortices among HIV-infected persons during a monetary decision-making task. Hum Brain Mapp 37:2455-2467

Montoya JL, Cattie J, Morgan E, Woods SP, Cherner M, Moore DJ, Atkinson JH, Grant I, Translational Methamphetamine Aids Research Center G (2016) The impact of age, HIV serostatus and seroconversion on methamphetamine use. Am J Drug Alcohol Abuse 42:168-177

Morgan EE, Iudicello JE, Weber E, Duarte NA, Riggs PK, DelanoWood L, Ellis R, Grant I, Woods SP (2012) Synergistic Effects of HIV Infection and Older Age on Daily Functioning. J Acquir Immune Defic Syndr 61:341-348

Must A, Szabó Z, Bódi N, Szász A, Janka Z, Kéri S (2006) Sensitivity to reward and punishment and the prefrontal cortex in major depression. J Affect Disord 90:209-215

Nagelkerke NJD (1991) A note on a general definition of the coefficient of determination. Biometrika 78:691-692

Navaline HA, Snider EC, Petro CJ, Tobin D, Metzger D, Alterman AI, Woody GE (1994) Preparations for AIDS vaccine trials. An automated version of the Risk Assessment Battery (RAB): enhancing the assessment of risk behaviors. AIDS Res Hum Retroviruses 10:S281-S283

Norman MA, Moore DJ, Taylor M, Franklin D, Jr., Cysique L, Ake C, Lazarretto D, Vaida F, Heaton RK, Group H (2011) Demographically corrected norms for African Americans and Caucasians on the Hopkins Verbal Learning Test-Revised, Brief Visuospatial Memory Test-Revised, Stroop Color and Word Test, and Wisconsin Card Sorting Test 64-Card Version. J Clin Exp Neuropsychol 33:793-804

Orsini CA, Hernandez CM, Bizon JL, Setlow B (2019) Deconstructing value-based decision making via temporally selective manipulation of neural activity: Insights from rodent models. Cogn Affect Behav Neurosci 19:459-476

Paolillo EW, Pasipanodya EC, Moore RC, Pence BW, Atkinson JH, Grelotti DJ, Grant I, Heaton RK, Moore DJ (2020) Cumulative burden of depression and neurocognitive decline among persons with HIV: a longitudinal study. JAIDS J Acquir Immune Defic Syndr 84

Park H, Yang J, Vassileva J, Ahn W-Y (2021) Development of a novel computational model for the Balloon Analogue Risk Task: The exponential-weight mean-variance model. J Math Psychol 102:102532

Patton JH, Stanford MS, Barratt ES (1995) Factor structure of the Barratt impulsiveness scale. J Clin Psychol 51:768-774

Paydary K, Mahin Torabi S, SeyedAlinaghi S, Noori M, Noroozi A, Ameri S, Ekhtiari H (2016) Impulsivity, Sensation Seeking, and Risk-Taking Behaviors among HIV-Positive and HIV-Negative Heroin Dependent Persons. AIDS Research and Treatment 2016:5323256-5323256

Rabkin JG (2008) HIV and depression: 2008 review and update. Curr HIV/AIDS Rep 5:163-171

Rippeth JD, Heaton RK, Carey CL, Marcotte TD, Moore DJ, Gonzalez R, Wolfson T, Grant I (2004) Methamphetamine dependence increases risk of neuropsychological impairment in HIV infected persons. J Int Neuropsychol Soc 10:1-14
Rogers RD, Everitt BJ, Baldacchino A, Blackshaw AJ, Swainson R, Wynne K, Baker NB, Hunter J, Carthy T, Booker E, London M, Deakin JFW, Sahakian BJ, Robbins TW (1999) Dissociable deficits in the decision-making cognition of chronic amphetamine abusers, opiate abusers, patients with focal damage to prefrontal cortex, and tryptophan-depleted normal volunteers: evidence for monoaminergic mechanisms. Neuropsychopharmacology 20:322-339

Rooney AS, Moore RC, Paolillo EW, Gouaux B, Umlauf A, Letendre SL, Jeste DV, Moore DJ (2019) Depression and aging with HIV: associations with health-related quality of life and positive psychological factors. J Affect Disord 251:1-7

Rubin LH, Maki PM (2019) HIV, depression, and cognitive impairment in the era of effective antiretroviral therapy. Curr HIV/ AIDS Rep 16:82-95

Rubin LH, Neigh GN, Sundermann EE, Xu Y, Scully EP, Maki PM (2019) Sex differences in neurocognitive function in adults with HIV: patterns, predictors, and mechanisms. Curr Psychiatry Rep 21:94

Saloner R, Cherner M, Grelotti DJ, Paolillo EW, Moore DJ, Heaton RK, Letendre SL, Kumar A, Grant I, Ellis RJ (2020a) Lower CSF homovanillic acid relates to higher burden of neuroinflammation and depression in people with HIV disease. Brain Behav Immun 90:353-363

Saloner R, Cysique LA (2017) HIV-associated neurocognitive disorders: a global perspective. J Int Neuropsychol Soc 23:860-869

Saloner R, Fields JA, Marcondes MCG, Iudicello JE, von Känel S, Cherner M, Letendre SL, Kaul M, Grant I, the Translational Methamphetamine ARCG (2020b) Methamphetamine and Cannabis: A Tale of Two Drugs and their Effects on HIV, Brain, and Behavior. J Neuroimmune Pharmacol

Smoski MJ, Lynch TR, Rosenthal MZ, Cheavens JS, Chapman AL, Krishnan RR (2008) Decision-making and risk aversion among depressive adults. J Behav Ther Exp Psychiatry 39:567-576

Soontornniyomkij V, Kesby JP, Morgan EE, Bischoff-Grethe A, Minassian A, Brown GG, Grant I (2016) Effects of HIV and methamphetamine on brain and behavior: evidence from human studies and animal models. J Neuroimmune Pharmacol 11:495-510

Strenziok M, Pulaski S, Krueger F, Zamboni G, Clawson D, Grafman J (2011) Regional brain atrophy and impaired decision making on the balloon analog risk task in behavioral variant frontotemporal dementia. Cogn Behav Neurol 24:59-67

Sundermann EE, Heaton RK, Pasipanodya E, Moore RC, Paolillo EW, Rubin LH, Ellis R, Moore DJ, Group H (2018) Sex differences in HIV-associated cognitive impairment. AIDS (london, England) 32:2719-2726

Thames AD, Streiff V, Patel SM, Panos SE, Castellon SA, Hinkin CH (2012) The role of HIV infection, cognition, and depression in risky decision-making. J Neuropsychiatry Clin Neurosci 24:340-348

Wallsten TS, Pleskac TJ, Lejuez CW (2005) Modeling behavior in a clinically diagnostic sequential risk-taking task. Psychol Rev 112:862-880

Wardle MC, Gonzalez R, Bechara A, Martin-Thormeyer EM (2010) Iowa Gambling Task performance and emotional distress interact to predict risky sexual behavior in individuals with dual substance and HIV diagnoses. J Clin Exp Neuropsychol 32:1110-1121

White TL, Lejuez CW, de Wit H (2007) Personality and gender differences in effects of d-amphetamine on risk taking. Exp Clin Psychopharmacol 15:599

Whiteside SP, Lynam DR (2001) The five factor model and impulsivity: Using a structural model of personality to understand impulsivity. Personality Individ Differ 30:669-689

Whittle EL, Fogarty AS, Tugendrajch S, Player MJ, Christensen H, Wilhelm K, Hadzi-Pavlovic D, Proudfoot J (2015) Men, 
depression, and coping: are we on the right path? Psychol Men Masculinity 16:426

Wilkinson G, Robertson G (2006) Wide Range Achievement Test-4 (WRAT-4). Psychological Assessment Resources Inc, Lutz, FL

Woods SP, Moore DJ, Weber E, Grant I (2009) Cognitive neuropsychology of HIV-associated neurocognitive disorders. Neuropsychol Rev 19:152-168

World Health Organization (1998) Composite Diagnositic International Interview (CIDI, version 2.1). World Health Organization: Geneva, Switzerland
Yu S, Shen Z, Lai R, Feng F, Guo B, Wang Z, Yang J, Hu Y, Gong L (2018) The Orbitofrontal Cortex Gray Matter Is Associated With the Interaction Between Insomnia and Depression. Front Psych 9:651-651

Publisher's Note Springer Nature remains neutral with regard to jurisdictional claims in published maps and institutional affiliations. 